\title{
3D Digital modeling for archeology using close range photogrammetry
}

\author{
Abbas Khalaf ${ }^{1,{ }^{*}}$, Tariq Ataiwe ${ }^{1}$, Israa Mohammed $^{1}$, and Ali Kareem ${ }^{1}$ \\ ${ }^{1}$ Building and Construction Engineering Department, University of Technology, Baghdad, Iraq
}

\begin{abstract}
This research is to evaluate the feasibility of applying three-dimensional modelling of the closerange photogrammetry in documenting archaeological monuments by using digital photogrammetry image processing software and digital consumer camera. The digital camera used was Nikon D3100, the processing software was (AgiSoft PhotoScan) and (ArcGIS, ArcScene extension). The study area was selected in the centre of Baghdad province by choosing one of the archeological monuments in it, namely the Abbasid alace. A set of camera locations represent the locations of the images, and as a result of the processing, 81 digital images were arranged in a sequence in which the results of this step were verified. The points cloud after processing were $1,082,617$ points. Six control points were selected, used as distances constrained. The validity of the fixed location of the points can be ascertained by checking the data. The program provide the error and accuracy for each image, where a total error in the scale bar was 0.005253 meters, a total error of marks points was 0.010957 meters and the accuracy for all six points was 0.005 meters.
\end{abstract}

\section{Introduction}

The importance of archaeological sites come from that it represents an important dimension of civilization and legacy, and as a means of identification for originated peoples and originality confirmation on the identity, and attracting source for tourism, and a means to communicate with the different communities by attract the interested researchers and scholars to these vestiges. Hence, the importance of preserving this heritage has emerged through sustainability documented in various ways and styles to bring out all the details and content of the simplified manner and attractive to the attention of the recipient and show the evident as it is [1,2].

he means of documentation of archaeological sites become more diverse, varied studies aimed at documenting archaeological sites, traditional studies which requires a long time and big effort or required unconventional expertise and skills at the same time a high cost, so it necessary to find ways to achieve a lowcost, ease to implement, with simple skills, less time than previous methods, new style to attract and interactive and is able to give a real impression, all of that can be found through the three-dimensional modeling with close range Photogrammetry[3,4].

Through the development of different technologies, operating systems and application systems, the users are provided with tools and means to easily handle the size of the information obtained from the authentication and treatment process. In order to reach the goal of the study, the research dealt with the construction of a threedimensional model of one known archaeological site and the definition of its history and its structure. The definition the photogrammetry, types and its divisions and the mechanism of work, and the possibilities of the analytical elements of features and acquire the necessary documentation of information through use of multiple images of the site.

Three-dimensional modelling from multiple images without using Ground Control Points, this can be done using a specialized software in the processing of multiple images and generating three-dimensional model (such as a Agisoft PhotoScan program) $[5,6]$.

\section{Research goals}

The aim of the research is to document the heritage buildings as an input to the process of preserving the heritage environment by presenting the different methods of documentation and finding the least complicated and least expensive way. This is done by presenting a three dimensional and interactive model including all the details. The feasibility of forming a database and information for all the effects as a basic reference for documentation and restoration with the possibility of the introduction of these data and models for display on the Internet and specifically to serve researchers and those interested in this area.

\section{Study area}

The study area was selected in the center of Baghdad province by choosing one of the archeological 
monuments in it, namely the Abbasid palace, as shown in Figure 1, within the coordinates below which dates back to the 5 th century $\mathrm{AH}$, which consists of two-storey building with internal dimensions (21.5 x $20 \mathrm{~m})$, each floor has many rooms and various uses with a large central courtyard and main entrance, we choose this archaeological site because it is one of the most important landmarks in Baghdad.

The coordinates of the site:

- WGS_1984_UTM_Zone_38N

- P1 = ( E= 442632.195, N=3689511.394 $) \mathrm{m}$

- $\mathrm{P} 2=(\mathrm{E}=442646.826, \mathrm{~N}=3689451.54) \mathrm{m}$

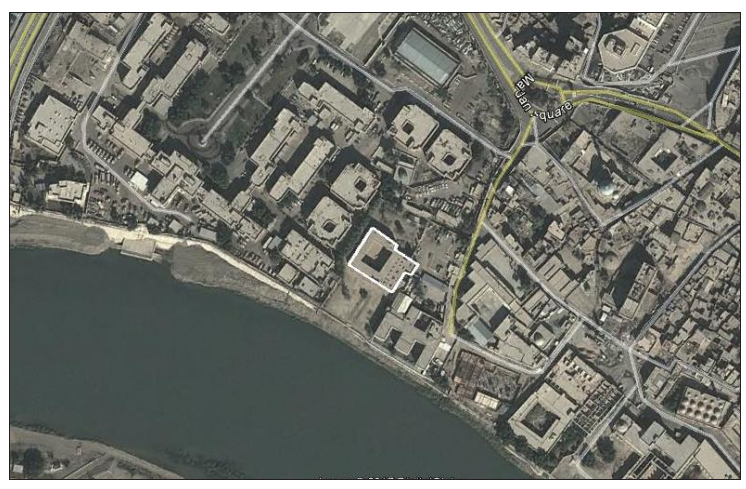

Fig. 1. Abbasid palace

\section{Research materials and methodology}

The Materials used in this research include the following:

1-A digital camera type Nikon D3100 For the purpose of taking pictures of the study site with different locations and angles.

2- A software package specializing in three-dimensional modeling and analysis of Close Range Photogrammetry (AgiSoft PhotoScan)

3 - Using ArcScene, one of the extensions of ArcGIS

- The methodology of the work can be summarized in the following igure (2) :

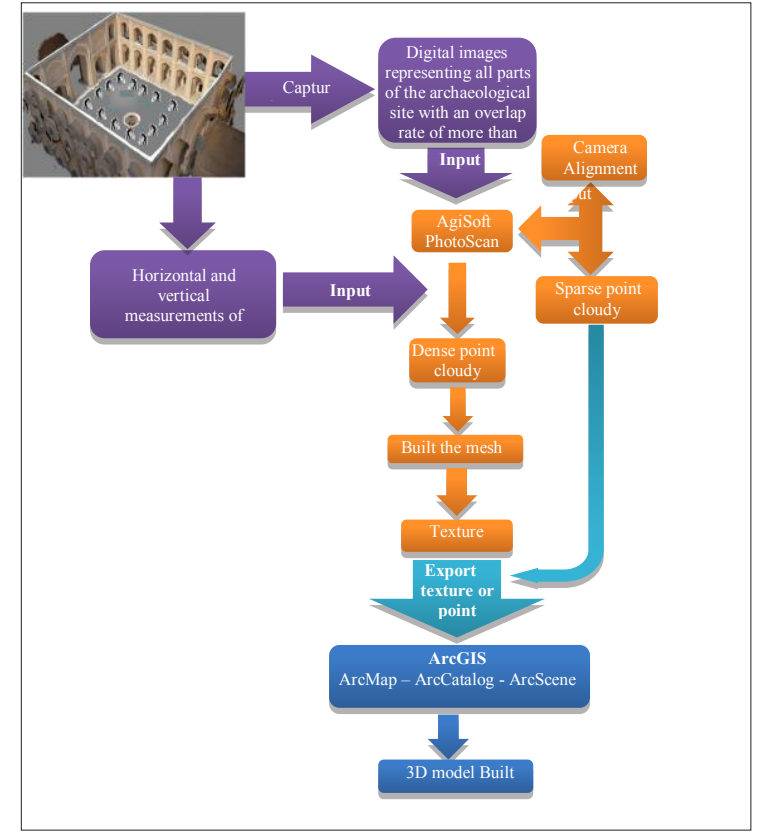

Fig. 2. The methodology flowchart

\section{Create a 3D model using Agisoft PhotoScan}

After collecting the images that were needed for the modeling process, we review images and remove distorted and unclear ones, which can be quickly identified before introducing to the program to get the results faster and better and then add the required images to the program and start the steps of treatment as follows:

- The first step is the process of organizing images and rearranging them to be sequential (Camera Alignment). In this step, the program will search for the common points in the images and work on matching them, in addition to finding the location of the camera and for each image as in Fig. 3 and Fig. 4 and finding the calibration parameters of the camera. A set of camera locations represent the locations of the images, and as a result of the processing, 81 digital images were arranged in a sequence in which the results of this step were verified.

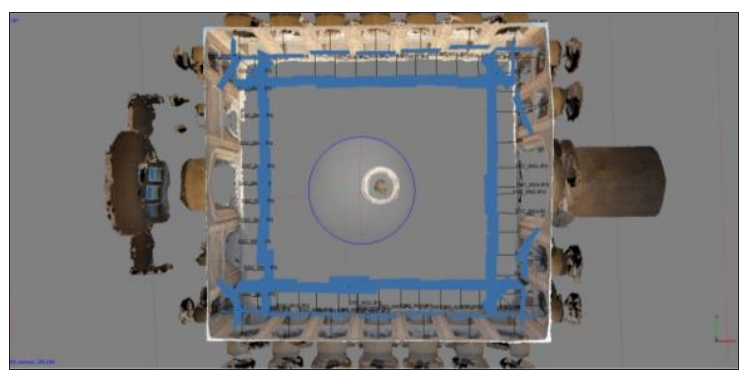

Fig. 3. Camera Alignment 


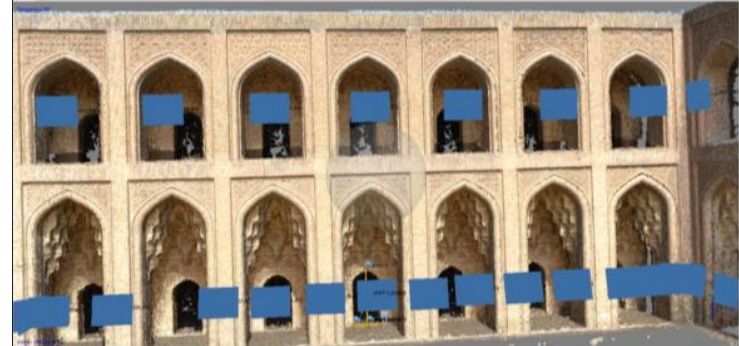

Fig. 4. Image capture locations on one of the sides for the corresponding side

This sparse point cloud figure (5), consisting of points greater than 1 million points $(1,082,617$ points), it is the result of image rearranging. It is not directly used in the creation of the $3 \mathrm{D}$ model, and can be exported for use in external programs. Example of using sparse point cloud is to be used in edit triangular or reference formats, and the camera location can be used to create $3 \mathrm{D}$ models.

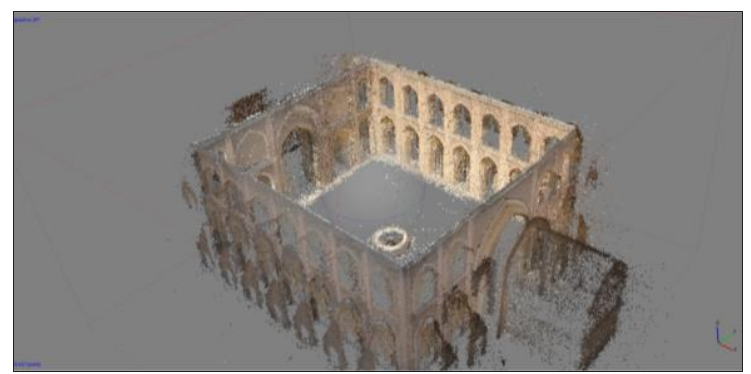

Fig. 5. Sparse point cloud

- In this step and through the tools available in the program we place the markers to fix the real scale - this is done by inserting distances measured between points as shown in figure (6). As the number of horizontal and vertical points increase, we can obtain better results. In our search, 6 points were selected used as distance constrained. The validity of the fixed location of the points can be ascertained by checking the data. The program provides the error and Accuracy for each image, where a total error in the scale bar was 0.005253 meters, a total error of marks points was 0.010957 meters and the accuracy for all six points was 0.005 meters.

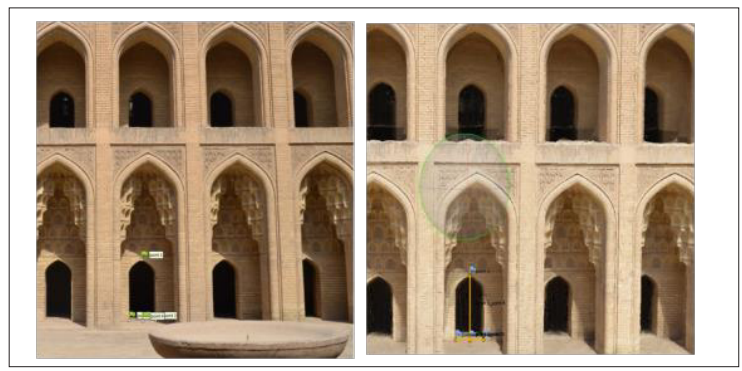

Fig. 6. The location of markers

- In this step, the dense point cloud as depicted in Figure (7) is constructed. The number of points formed is more than thirty-four million points $(34,813,976$ points). It is constructed depending on the locations of the cameras. The program creates a dense cloud that can be edited or classified before being exported and processed to create the network for the three-dimensional model.

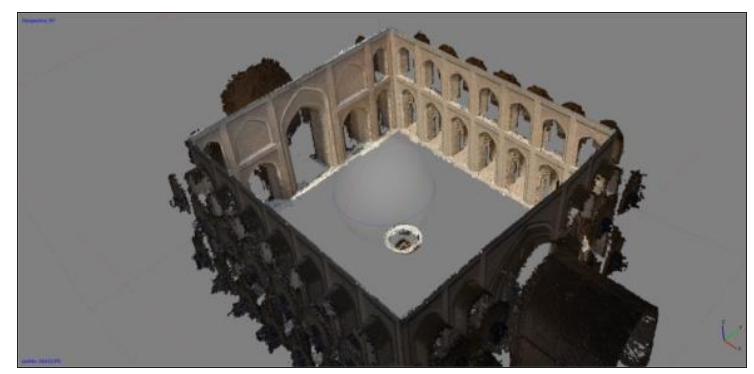

Fig. 7. Dense point cloud

- The next step is to build the mesh as shown in igure (8), where the program creates a three-dimensional mesh representing the body's surface based on the thick point cloud created in the previous phase. In addition, it is possible to create a geometric model and a polygonal network faster by creating it on the scattered points cloud alone.

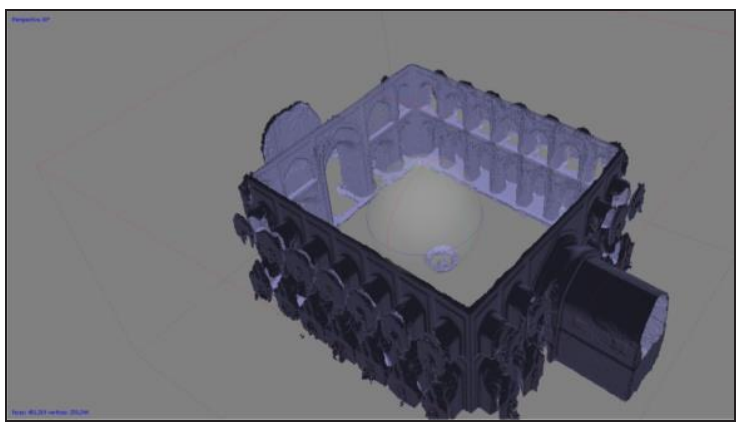

Fig. 8. Built the mesh

- After the construction mesh the construction of the outer texture as shown in figure (9) and igure (10) or generation of orthophoto can be completed [7].

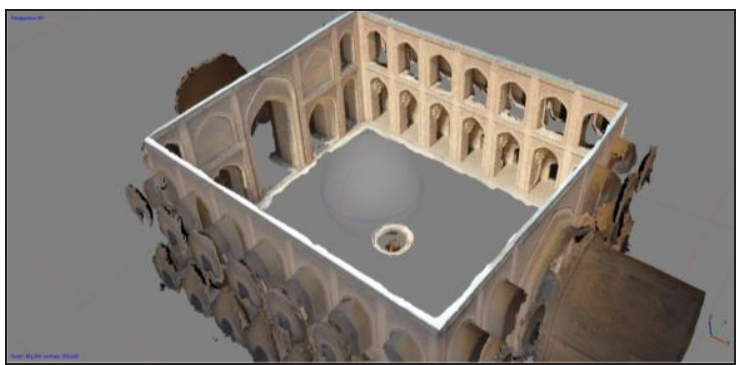

Fig. 9. Outer texture 


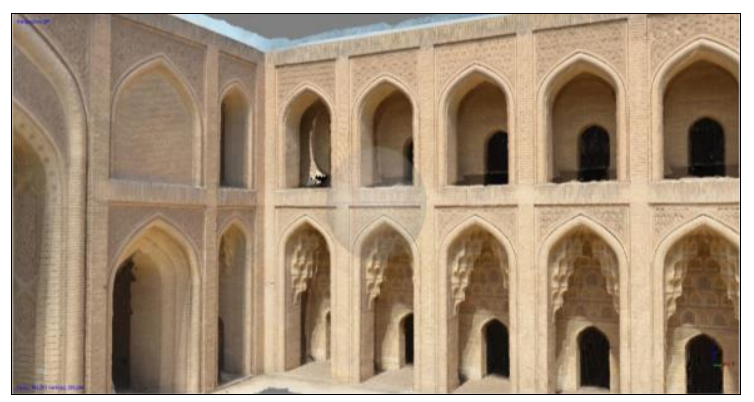

Fig. 10. Final outer texture

\section{Create a 3D model using ArcGIS}

Using ArcGIS 10.3 with its main extensions: ArcMap, ArcCatalog, ArcScene

After exporting the points from the AgiSoft PhotoScan program in text file format, we use ArcMap, import the points file figure (3-16), and convert the points into a shapefile figure (3-17) which can be used, and through the $3 \mathrm{D}$ data for the points $(\mathrm{X}, \mathrm{Y}, \mathrm{Z})$ to build a $3 \mathrm{D}$ model in the ArcScene format (3-18)

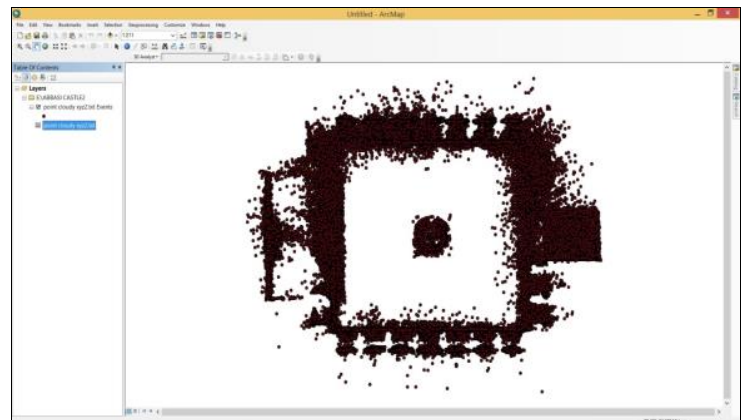

Fig. 11. Points in ArcMap

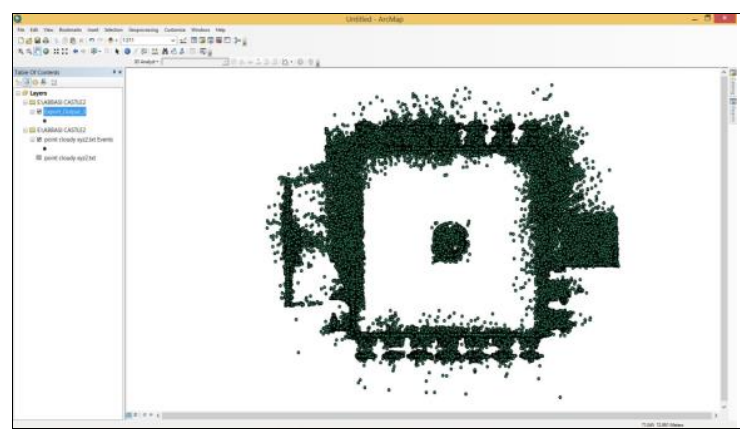

Fig. 12. Shapefile

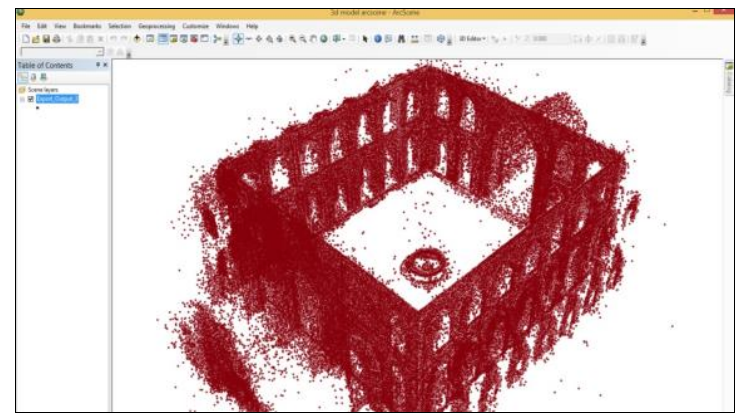

Fig. 13. 3D Points in the ArcScene format

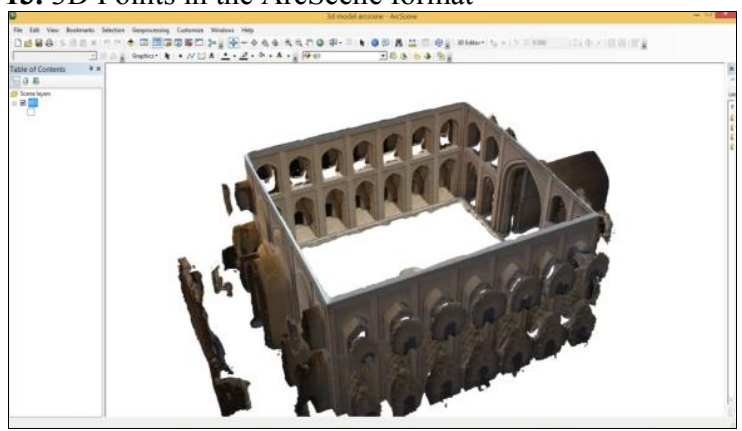

Fig. 14. Build a 3D model in the ArcScene

\section{Conclusions:}

Based on the results of the study, we can summarize the conclusions as follows:

- Development of imaging equipment, image capture and processing software has helped the possibility of building full-scale digital engineering models using lowcost digital cameras and personal computer software,

- The methodology used in this research saves money and time and does not require special imaging expertise compared with laser scanning, the price of the camera did not exceed 500 USD, the processing time was approximately 24 hours and the photos gathered by nonprofessional photographer, but required to follow the guidelines of the work plan in the manner of taking multiple images for the purposes of photogrammetry and is especially effective in areas with limited spaces.

\section{Recommendations:}

- The need to use of electronic means in the process of electronic documentation to obtain accurate results. - Perform documentation using 3D modeling for all sites and incorporate them into a comprehensive database.

- Increasing awareness of the importance of heritage as a cultural and tourist source.

\section{References}

1. F. Saleh, R. Bahgat, In 29th CAA conf. Proc. (2001).

2. Paul R.W., Bon A. D., Benjamin E. W., Elements of photogrammetry: with applications in GIS (McGraw-Hill, 2014)

3. Mario A. G., Basics of geomatics (Springer, 2010).

4. H. Lin, J. Zhu, B. Xu, W. Lin, Y. Hu, J. Lee, S. Zlatanova $3 D$ Geo-Information Sciences (Springer, 2009).

5. Stephen R. Design and Implementation of a Spatially Enabled Panoramic Virtual Reality Prototype (UNB, 2002).

6. G. Olga, K. Dimitrios, K. Georgios, P. Petros, In 29th CAA conf. Proc. (2001).

7. L. Agisoft, Agisoft PhotoScan user Manual (Agisoft LLC, 2016) 